\title{
A modified AUGIS Delphi process to establish future research priorities in benign upper gastrointestinal surgery
}

\section{Authors}

Michael SJ Wilson ${ }^{1}$, Vaughan-Shaw $\mathrm{P}^{2}$, Boyle $\mathrm{C}^{3}$, Yong $\mathrm{GL}^{4}$, Oglesby $\mathrm{S}^{5}$, Skipworth $\mathrm{R}^{6}$, Lamb $\mathrm{P}^{6}$, Griffiths EA $\mathrm{T}^{7}$ and Attwood SEA ${ }^{8}$ on behalf of AUGIS

\section{$\underline{\text { Affiliations }}$}

${ }^{1}$ Consultant Surgeon, Department of General Surgery, Forth Valley Royal Hospital, Larbert, FK5 4WR

${ }^{2}$ Specialty Registrar, Department of Colorectal Surgery, Western General Hospital, Edinburgh, EH4 2XU

${ }^{3}$ Graduate School of Education, University of Exeter

${ }^{4}$ Specialty Registrar, Department of Radiology, Royal Infirmary of Edinburgh, EH16 4SA

${ }^{5}$ Consultant Upper GI Surgeon, Department of Upper GI Surgery, Ninewells Hospital, Dundee, DD2 1SY

${ }^{6}$ Consultant Upper GI Surgeon, Department of Upper GI Surgery, Royal Infirmary of Edinburgh, EH16 4SA

${ }^{7}$ Consultant Upper GI Surgeon, Department of Upper GI Surgery, Queen Elizabeth Hospital, Birmingham, B15 2TH

${ }^{8}$ Professor of Health Services Research, Department of Health Services Research, Durham University, Durham, DH1 3RW

Corresponding author

Michael SJ Wilson

Consultant General and Upper GI surgeon

Department of General Surgery

Forth Valley Royal Hospital

Larbert

FK5 4WR

Tel: 01324566000

Email: michaelwilson3@nhs.net

Short title

Research priorities in benign Upper GI surgery

Keywords

Research priorities, upper gastrointestinal surgery

All authors declare no conflicts of interest

Word count $=2,257$ 


\section{Abstract}

\section{Background}

The aim of our study was to use a modified Delphi process to determine the research priorities among benign upper gastrointestinal (UGI) surgeons in the United Kingdom.

\section{Methods}

Delphi methodology may be utilised to develop consensus opinion amongst a group of experts. Members of the Association of Upper Gastrointestinal Surgeons of Great Britain and Ireland were invited to submit individual research questions via an online survey (phase I). Two rounds of prioritisation by multidisciplinary expert healthcare professionals (phase II and III) were completed to determine a final list of high priority research questions.

\section{Results}

427 questions were submitted in phase I and 51 with a benign UGI focus were taken forward for prioritisation in phase II. 28 questions were ranked in phase III. A final list of 11 high priority questions had an emphasis on acute pancreatitis, Barrett's oesophagus and benign biliary disease.

\section{Conclusion}

A modified Delphi process has produced a list of 11 high priority research questions in benign UGI surgery. Future studies and awards from funding bodies should reflect this consensus list of prioritised questions in the interest of improving patient care and encouraging collaborative research. 


\section{Introduction}

The scope of benign upper gastrointestinal (UGI) surgery is broad and includes the management of common conditions such as; cholelithiasis, pancreatitis, Barrett's oesophagus and gastro-oesophageal reflux disease. Other conditions may be encountered less frequently; paraoesophageal hernia, gastric volvulus, achalasia and oesophageal dysmotility. Not all of these conditions require operative intervention but each of them may require input from an UGI surgeon with a benign subspecialty interest. An evidence based approach is the ideal way to manage patients with these conditions, but a significant proportion of benign UGI practice lacks an evidence base.

A modified Delphi process can be used to develop a list of priorities by consensus from a group of experts. This has been successfully utilised in colorectal surgery (1), orthopaedics (2) plastic surgery (3) and hepatobiliary surgery (4). This approach in determining research priorities improves efficiency and adds greater value to those who fund benign UGI surgery research (5).

To our knowledge no attempt has previously been made to determine the future research priorities in benign UGI surgery. The aim of our study was to undertake a modified Delphi process to determine the research priorities in benign UGI surgery. 


\section{Material and Methods}

A three-phased modified Delphi process was undertaken (Figure 1). This included two distinct phases of prioritisation by expert multidisciplinary stakeholders utilising established methodology, as previously described for a number of clinical projects $(1,6,7)$. Stakeholders were asked to submit questions and, thereafter, prioritise their responses based upon their own perceived clinical need. During the prioritisation phases (II and III), only complete submissions where all questions were ranked were included in the analysis.

Phase I

Experts were recruited from the Association of Upper GI Surgeons of Great Britain and Ireland (AUGIS) membership, which includes medical professionals and members of the wider multidisciplinary team such as research nurses, dietitians and specialist nurses. Members were invited by email to submit research questions across the entire spectrum of UGI and hepato-pancreato-biliary (HPB) surgery (including both benign and malignant conditions) via an online survey (http://surveymonkey.com). The social media platform Twitter was also used to broaden the awareness of the Delphi process amongst interested stakeholders. There was no limit on the number of research questions that an individual could submit. The survey was open to submissions for 3 month, with three email reminders sent to the AUGIS membership during this period.

Submitted questions were collated and then grouped into four categories: 1) HPB; 2) Benign UGI; 3) Malignant oesophagogastric (OG); and 4) Bariatric and metabolic surgery. Any disagreements regarding categorisation were resolved by consensus. 
To assess category (2) above, a benign UGI surgery steering committee was formed.

Duplicate questions were removed. Questions with a similar theme were altered by consensus agreement of the steering committee. Care was taken not to alter the meaning of the reviewed questions.

Phase II

Benign UGI surgery research questions were prioritised by AUGIS members by email invitation with a link to an online survey (Google forms). Twitter was again used to highlight the prioritisation process amongst interested stakeholders. The survey contained all of the Benign UGI surgery research questions and respondents were asked to prioritise each question using a Likert scale ( 1 - lowest priority to 5 - highest priority). The survey remained open to submissions for 11 weeks with three email reminders sent to AUGIS members. The results were reviewed by the steering committee and a 'cut-off' point agreed by consensus based on a mean score $\geq 3.0$ following prioritisation for inclusion in the final round of prioritisation.

Phase III

A final round of prioritisation was performed after AUGIS members were again invited by email and Twitter to follow a link to a Google forms survey and prioritise the questions using the same Likert scale as in Phase II. The survey remained open for 7 weeks and three email reminders were sent. Results were reviewed by the steering committee to identify the final list of prioritised questions. The criteria for inclusion in the final list of research priorities was a mean score of $\geq 3.5$, a Likert score of $4-5$ by $>50 \%$ respondents and a Likert score of 1 2 by $<20.0 \%$ respondents. 
Steering committee

The benign UGI surgery steering committee consisted of one Upper GI senior surgical trainee (MW), five consultant upper GI surgeons (SO, EG, RS, SA and PL) and lay representation $(\mathrm{CB}, \mathrm{GY})$. The overall role of the steering committee was to ensure relevance of the submitted questions from both a clinical and patient perspective and to provide consensus agreement. 


\section{Results}

Four hundred and twenty seven research questions were submitted by 140 AUGIS members in Phase I, representing $47.6 \%$ of the membership (Figure 2). Of those responding, a subspecialisation benign UGI surgery interest was declared by 68 (48.6\%).

Once duplicated and similar questions were reviewed and amended or removed by consensus agreement, 51 questions were moved forward for prioritisation in phase II. Sixtynine stakeholders voluntarily prioritised the questions in phase II. An analysis of the prioritisation was performed by the steering committee and consensus reached regarding a cut-off for inclusion (mean $\geq 3.0$ ) in phase III.

Twenty-eight questions were included in the final phase of prioritisation and 65 surgical stakeholders took part. Following review by the steering committee with consensus agreement on the criteria for inclusion on the final list of clinical priorities as detailed in the methods section, 11 questions were included on the final list of benign UGI surgery questions with high research priority (Figure 3). Our list of prioritised questions focused on the following themes;

\section{1) Acute pancreatitis}

\section{2) Barrett's oesophagus (BE)}

\section{3) Benign biliary disease}

4) Other - achalasia, GERD, hiatus hernia

The questions which failed to make the final list of research priorities from phase III can be seen in Appendix 1. 


\section{Discussion}

This study has produced a list of 11 high priority research questions relevant to the surgical subspecialty of benign UGI surgery using a modified Delphi process. To our knowledge this is the first time that such a project has been undertaken in the field of benign Upper GI surgery. This study was undertaken as part of a wider project to determine the research priorities in the broader specialty of Upper GI surgery (which also incorporated the subspecialty interests of bariatric and metabolic surgery, HPB (4) and malignant oesophagogastric surgery).

From our list of prioritised questions there is an emphasis on focusing future research on the management of acute pancreatitis, Barrett's oesophagus (BE) and benign biliary disease. Our final list also included questions relating to the management of GERD, hiatus hernia repair and achalasia.

The emphasis on acute pancreatitis may be unsurprising as it is frequently encountered during the acute general surgery take and can lead to significant morbidity and difficult management decisions (8). Three questions relating to acute pancreatitis ranked sufficiently to be included in our final list of research priorities. Hospital readmissions as a result of pancreatitis can be as high as $17 \%$ (9). Recent evidence suggests an increased incidence of pancreatitis among younger patients, reduced incidence in older patients with the most common aetiologies being biliary disease and alcohol related (10). A recently published trial may have already addressed the question relating to whether patients with idiopathic 
pancreatitis benefit from a laparoscopic cholecystectomy (11). The authors concluded that laparoscopic cholecystectomy if effective for the treatment of recurrent idiopathic pancreatitis, although the methodology has subsequently been criticised $(12,13)$.

$B E$ contributes three questions to our final list of research priorities. The reasons for this are unclear. One explanation may be that the majority of UGI surgeons encounter this condition in their endoscopic practice, and interest in this condition is likely to be higher amongst those with a malignant OG interest. Interest in BE may have been heightened by the recent publication of the AspECT trial which reported improved outcomes in those who receive a combination of high dose PPI aspirin (14). The inclusion of the questions relating the true risk of progression and the surveillance programme for Barrett's oesophagus are also unexpected as they are likely to be answered upon completion of the BOSS trial (15). The BOSS trial is a large UK randomised trial of regular surveillance endoscopy versus on demand endoscopies in patients with BE and is due to report in 2022. Equally, the inclusion of the question that relates to the natural history of small stones in the common bile duct may also be addressed in the Sunflower Study (16). The Sunflower Study is a large pragmatic UK randomised trial of pre-operative MRCP versus no imaging in patients with low to medium risk of CBD stones have either emergency or elective laparoscopic cholecystectomy. It is currently recruiting in the pilot phase.

The Delphi process of research questions importance to UK surgeons does not measure the feasibility of studies, the costs and how they would be funded, the likelihood that a study design would effectively or adequately answer the clinical question, nor does the list take in to account studies that are already underway that would address the questions posed. For 
example the BOSS trial is prospectively following 3,500 patients with Barrett's oesophagus and looking at the relative value of surveillance every 2 years versus at need, and the Aspect trial has just reported the outcome of 2,500 patients with Barrett's oesophagus where the dose of PPI with or without aspirin has been assessed for cancer prevention. In both studies the true underlying disease progression of Barrett's to cancer will be available and in a cohort of 6,000 patients with more than 7 years prospective follow up. These studies will answer the related questions highlighted by the Delphi process $\left(5^{\text {th }}\right.$ and $6^{\text {th }}$ questions in Figure 3).

The frequency of achalasia is relatively low and so it would be highly unlikely that a UK trial could answer the question ( $9^{\text {th }}$ in Fig 3 ) on whether laparoscopic Heller's cardiomyotomy with antireflux procedure is superior to per-oral endoscopic myotomy (POEM) but there is already a European multi-national study underway looking at these two options (17). One of the great difficulties of comparing procedures is the variation in technical training, learning curve and the quality of operative skills in different countries and in different surgical specialties. Often, the best therapy may not be one particular operation or procedure but the one that the institution or clinician is trained to perform and most familiar with.

There has long been a debate regarding the specific technical details regarding antireflux surgery. At its most basic level this could be about whether a Nissen fundoplication is better than a partial fundoplication? However, partial wraps can be anterior or posterior, and even amongst these broad categories significant variations in technique exist, including formation of the wrap, number of sutures, type of sutures, division of the short gastric vessels and closure of the hiatus to name just a few. In relation to the $10^{\text {th }}$ question in 
Figure 3 it is the generally held view that the most appropriate anti reflux operation is probably the one the individual surgeon and institution has the most experience in performing. Prospective research to prove or disprove this is complicated by generalisability of any particular clinical trial. For many years the indications for anti reflux surgery have not been standardised, but recent guidelines may help to address this in the future (18).

A number of questions failed to make the final list of prioritised questions (Appendix1) and the reasons are likely to be multifactorial. The questions posed may be due to be answered by current studies. An example of this would be 'What is the optimal management of asymptomatic choledocholithiasis?' which is being addressed in the Sunflower study. Alternatively, the proposed question may have already been answered. An example of this would include 'what is the optimal time to perform an emergency laparoscopic cholecystectomy for acute cholecystitis?' and 'is emergency laparoscopic cholecystectomy cost effective?' (19-22).

In the final round of prioritisation, all responses were submitted by surgeons. This is a limitation of our study. A broader range of contributions from the Upper GI multidisciplinary team (radiologists, dietitians, specialist nurses) may have been desirable. Twitter was used to publicise the existence of the survey, and therefore the survey was in the public domain and open to submissions from lay individuals, patients and family members. Unfortunately, no submissions were received from non-healthcare professionals. We did have lay representation on the committee, both of whom have prior experience of Delphi methodology. CB and GY were involved in the discussion and agreement upon methodology in phase II and III of the study. 
Previous Delphi processes in other specialties reported response rates ranging from 11 to $25 \%$. Our response rate in phase I across all specialties was $47.6 \%$ and this should therefore be considered as sufficient engagement from the AUGIS membership. The list of prioritised research questions will be shared with funding bodies. The expectation is that our list of research questions will provide a focus of future research topics and be a useful resource for research grant and clinical trial applications. Further, AUGIS members who contributed to this study at any point from phase I to II may become motivated to undertake future research in to some of the questions identified by this consensus agreed Delphi process.

In summary, our modified Delphi process has produced a list of questions that have been deemed by consensus amongst UK benign UGI surgical specialists to have the highest research priority in the field of OG cancer surgery. There is an emphasis on acute pancreatitis, BE and benign biliary disease. Future research projects should seek to address these questions as well as to engage improved patient and public involvement. 


\section{Acknowledgments}

The benign UGI steering committee wish to formally thank AUGIS and BOMSS for their support with this study. In particular, the administrative support provided by Nichola Bartlett was fundamental throughout the Delphi process. 


\section{References}

1. Tiernan J, Cook A, Geh I, George B, Magill L, Northover J, et al. Use of a modified Delphi approach to develop research priorities for the association of coloproctology of Great Britain and Ireland. Colorectal Dis. 2014;16(12):965-70.

2. Eubank BH, Mohtadi NG, Lafave MR, Wiley JP, Bois AJ, Boorman RS, et al. Using the modified Delphi method to establish clinical consensus for the diagnosis and treatment of patients with rotator cuff pathology. BMC Med Res Methodol. 2016;16:56.

3. Henderson J, Reid A, Jain A. Use of a modified BAPRAS Delphi process for research priority setting in Plastic Surgery in the UK. J Plast Reconstr Aesthet Surg. 2018;71(12):167981.

4. Knight SR, Pathak S, Christie A, Jones L, Rees J, Davies H, et al. Use of a modified Delphi approach to develop research priorities in HPB surgery across the United Kingdom. HPB (Oxford). 2019.

5. Chalmers I, Bracken MB, Djulbegovic B, Garattini S, Grant J, Gulmezoglu AM, et al. How to increase value and reduce waste when research priorities are set. Lancet. 2014;383(9912):156-65.

6. Stewart RJ, Caird J, Oliver K, Oliver S. Patients' and clinicians' research priorities. Health Expect. 2011;14(4):439-48.

7. Burt CG, Cima RR, Koltun WA, Littlejohn CE, Ricciardi R, Temple LK, et al. Developing a research agenda for the American Society of Colon and Rectal Surgeons: results of a delphi approach. Dis Colon Rectum. 2009;52(5):898-905.

8. Goldacre MJ, Roberts SE. Hospital admission for acute pancreatitis in an English population, 1963-98: database study of incidence and mortality. BMJ. 2004;328(7454):14669.

9. Garg SK, Campbell JP, Anugwom C, Wadhwa V, Singh R, Gupta N, et al. Incidence and Predictors of Readmissions in Acute Pancreatitis: A Nationwide Analysis. Pancreas. 2018;47(1):46-54.

10. Garg SK, Sarvepalli S, Campbell JP, Obaitan I, Singh D, Bazerbachi F, et al. Incidence, Admission Rates, and Predictors, and Economic Burden of Adult Emergency Visits for Acute Pancreatitis: Data From the National Emergency Department Sample, 2006 to 2012. J Clin Gastroenterol. 2019;53(3):220-5.

11. Raty S, Pulkkinen J, Nordback I, Sand J, Victorzon M, Gronroos J, et al. Can Laparoscopic Cholecystectomy Prevent Recurrent Idiopathic Acute Pancreatitis?: A Prospective Randomized Multicenter Trial. Ann Surg. 2015;262(5):736-41.

12. Mentula P. Effect of Laparoscopic Cholecystectomy After Idiopathic Acute Pancreatitis May Be Overestimated. Ann Surg. 2017;266(6):e77-e8.

13. Said M, Rongen APM. Can Laparoscopic Cholecystectomy Prevent Recurrent Idiopathic Acute Pancreatitis? Ann Surg. 2017;266(6):e94-e5.

14. Jankowski JAZ, de Caestecker J, Love SB, Reilly G, Watson P, Sanders S, et al. Esomeprazole and aspirin in Barrett's oesophagus (AspECT): a randomised factorial trial. Lancet. 2018;392(10145):400-8.

15. Old O, Moayyedi P, Love S, Roberts C, Hapeshi J, Foy C, et al. Barrett's Oesophagus Surveillance versus endoscopy at need Study (BOSS): protocol and analysis plan for a multicentre randomized controlled trial. J Med Screen. 2015;22(3):158-64.

16. A randomised controlled trial to establish the clinical and cost effectiveness of expectant management versus pre-operative imaging with magnetic resonance cholangio- 
pancreaticogram (MRCP) in patients with symptomatic gallstones undergoing laparoscopic cholecystectomy at low or moderate risk of common bile duct stones: The Sunflower Study [Available from: http://www.isrctn.com/ISRCTN10378861.

17. Endoscopic Versus Laparoscopic Myotomy for Treatment of Idiopathic Achalasia: A Randomized, Controlled Trial (POEM) [Available from:

https://clinicaltrials.gov/ct2/show/NCT01601678.

18. Pauwels A, Boecxstaens V, Andrews CN, Attwood SE, Berrisford R, Bisschops R, et al. How to select patients for antireflux surgery? The ICARUS guidelines (international consensus regarding preoperative examinations and clinical characteristics assessment to select adult patients for antireflux surgery). Gut. 2019.

19. Wiggins T, Markar SR, MacKenzie H, Faiz O, Mukherjee D, Khoo DE, et al. Optimum timing of emergency cholecystectomy for acute cholecystitis in England: population-based cohort study. Surg Endosc. 2019.

20. Zafar SN, Obirieze A, Adesibikan B, Cornwell EE, 3rd, Fullum TM, Tran DD. Optimal time for early laparoscopic cholecystectomy for acute cholecystitis. JAMA Surg.

2015;150(2):129-36.

21. Cao AM, Eslick GD, Cox MR. Early laparoscopic cholecystectomy is superior to delayed acute cholecystitis: a meta-analysis of case-control studies. Surg Endosc.

2016;30(3):1172-82.

22. Sutton AJ, Vohra RS, Hollyman M, Marriott PJ, Buja A, Alderson D, et al. Costeffectiveness of emergency versus delayed laparoscopic cholecystectomy for acute gallbladder pathology. Br J Surg. 2017;104(1):98-107. 